\title{
Novel PTRF mutation in a child with mild myopathy and very mild congenital lipodystrophy
}

\author{
Anna Ardissone ${ }^{1}$, Cinzia Bragato ${ }^{2}$, Lorella Caffi ${ }^{3}$, Flavia Blasevich² ${ }^{2}$ Sabrina Maestrini ${ }^{4}$, Maria Luisa Bianchi ${ }^{5}$, \\ Lucia Morandi $^{2}$, Isabella Moroni ${ }^{1}$ and Marina Mora ${ }^{2,6^{*}}$
}

\begin{abstract}
Background: Mutations in the PTRF gene, coding for cavin-1, cause congenital generalized lipodystrophy type 4 (CGL4) associated with myopathy. In CGL4, symptoms are variable comprising, in addition to myopathy, smooth and skeletal muscle hypertrophy, cardiac arrhythmias, and skeletal abnormalities. Secondary features are atlantoaxial instability, acanthosis nigricans, hepatomegaly, umbilical prominence and metabolic abnormalities related to insulin resistance, such as diabetes mellitus, hyperlipidemia and hepatic steatosis.
\end{abstract}

Case presentation: We describe a 3 year-old child of Moroccan origin with mild muscle phenotype, mainly characterized by mounding, muscle pain, hyperCKemia and mild caveolin 3 reduction on muscle biopsy. No CAV3 gene mutation was detected; instead we found a novel mutation, a homozygous single base pair deletion, in the PTRF gene. Only after detection of this mutation a mild generalized loss of subcutaneous fat, at first underestimated, was noticed and the diagnosis of lipodystrophy inferred.

Conclusions: The PTRF gene should be investigated in patients with hyperCKemia, mild myopathy associated with spontaneous or percussion-induced muscle contractions like rippling or mounding, and no CAV3 mutation. The analysis should be performed even if cardiac or metabolic alterations are absent, particularly in young patients in whom lipodystrophy may be difficult to ascertain.

Keywords: Congenital generalized lipodystrophy type 4, Cavin-1, PTRF/CAVIN, Muscle mounding, HyperCKemia

\section{Background}

Congenital generalized lipodystrophies (CGLs) are autosomal recessive disorders characterized by almost complete absence of body fat. Various gene defects may cause the condition. Mutations in a new gene, PTRF (polymerase I and transcript release factor), coding for cavin-1, have recently been found to cause CGL type 4 (CGL4) associated with muscle disease [1-3].

Cavin-1 is an abundant protein component of caveolae, and member of a family of related proteins that form a caveola- associated multiprotein complex. The complex also contains serum deprivation response (SDR)/cavin-2, SDR-related gene product that binds to $C$ kinase

\footnotetext{
* Correspondence: mmora@istituto-besta.it

${ }^{2}$ Neuromuscular Diseases and Neuroimmunology Unit, Fondazione IRCCS

Istituto Neurologico C. Besta, Milano, Italy

"Muscle Cell Biology Lab, Fondazione IRCCS Istituto Neurologico "C. Besta",

Via Temolo 4, 20126, Milano, Italy

Full list of author information is available at the end of the article
}

$(\mathrm{SRBC}) /$ cavin-3, and muscle-restricted coiled-coil protein (MURC)/cavin-4 [4-8].

This complex can constitutively assemble in cytosol and associate with caveolin at plasma membrane caveolae. Cavin-1, but not other cavins, can induce caveola formation in a heterologous system and is required for recruitment of the cavin complex to caveolae [9]. Absence of cavin-1 leads to global loss of caveolae, and markedly diminished expression of all three caveolin protein isoforms, while caveolin mRNA levels are normal or above normal [10].

Cavin-1-knockout mice are viable and of normal weight but have high circulating triglycerides, low adipose tissue mass, glucose intolerance, and hyperinsulinemia (lipodystrophic phenotype).

Few PTRF mutations have so far been reported in humans [1-3,11,12]. We describe a 3-year-old boy with mild muscular phenotype in whom lipodystrophy was diagnosed only after detection of a novel homozygous mutation in the PTRF gene. 


\section{Case presentation}

The boy is the fourth child of healthy consanguineous (first cousins) parents of Moroccan origin. A sister and brother are in good health but the other sister died 6 hours after birth. Family history is negative for neuromuscular diseases.

The patient was born after an uncomplicated pregnancy with APGAR 8 at 1 minute and 9 at 5 minutes. He was noticed to have supinated left foot varus, mild dysmorphic features and reduced motility without hypotonia. Brain and renal ultrasound, EEG, and karyotype were normal. Normal psychomotor development was reported during the first year, but by $18-20$ months he had clumsy gait, muscle pain in the lower limbs, mild fatigability and elevated muscle enzymes (CK 1179-1589 IU/L, normal 24-195; LDH 446-697 IU/L, normal 230-480); ECG and thyroid function tests were normal.

When next seen at 3 years 4 months, the boy had a protruding abdomen, a marked umbilical prominence, mild generalized loss of subcutaneous fat and no acanthosis nigricans (Figure 1); his weight was $13 \mathrm{~kg}$ (3rd to 10th centile), height $94 \mathrm{~cm}$ (50th centile). Neurological examination showed normal cranial nerves, normal tone, mild axial weakness, with difficulty in sitting from supine position, but normal strength limb muscles, hypertrophic buttock and lower limb muscles (Figure 1), reduced lower limb tendon reflexes, lumbar lordosis, supinated left foot varus and clumsy gait. The patient was able to get up from the chair and to stand up from the floor without support. There was also marked and rapid percussion-induced muscle contraction and mounding in upper and lower limbs.

Cognitive development was normal. Muscle enzymes were elevated: CK 963 (normal<195 IU/L); LDH 767 (normal $<480 \mathrm{IU} / \mathrm{L})$; AST 45 (normal $<41 \mathrm{IU} / \mathrm{L})$; ALT 50 (normal < $37 \mathrm{IU} / \mathrm{L}$ ); aldolase 19.2 IU/L (normal < 7.6).

Brain and cervical spinal cord MRI were normal; EMG revealed mild myopathic alterations and absence of myotonic discharges. Lower limb CT showed muscle hypertrophy and marked loss of subcutaneous fat (Figure 2). ECG was normal; abdominal ultrasonography was normal: in particular liver and spleen enlargements were excluded.

Muscle biopsy, after informed parental consent, showed moderate variation in fiber size, fibers with central nuclei, and mild endomysial fibrosis (Figure 3B). Muscle immunostaining showed mildly reduced and variable expression between fibers of caveolin-3 (Figure 3A), but CAV3 gene sequencing failed to reveal any pathogenic mutation.

On sequencing the PTRF gene, a novel homozygous single base pair deletion, c.947delA was found (Figure 3D); the same mutation was present heterozygously in each

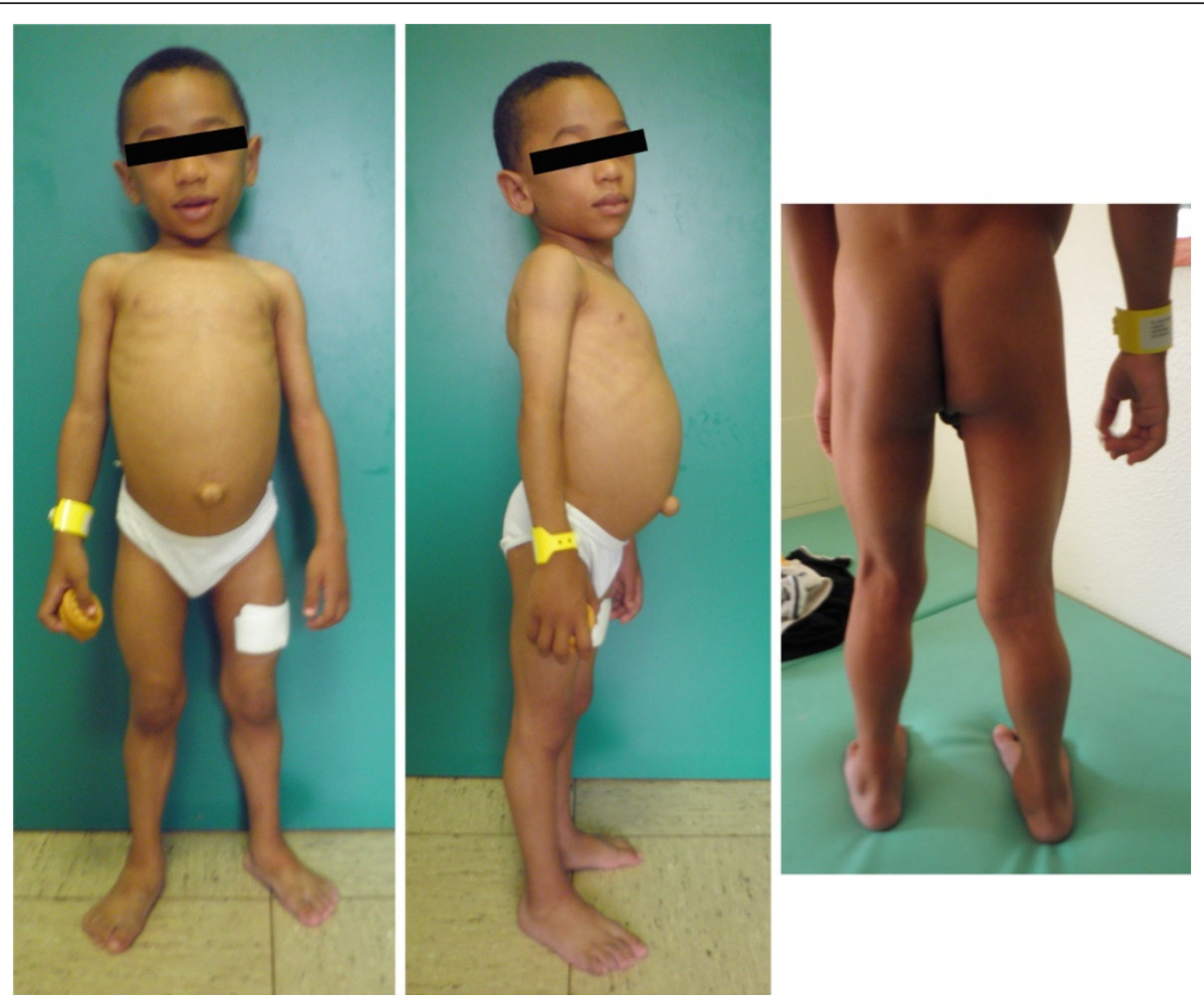

Figure 1 Photographs of our patient. Protruding abdomen, marked umbilical prominence, mild generalized loss of subcutaneous fat, and hypertrophic buttock and lower limb muscles are shown. 

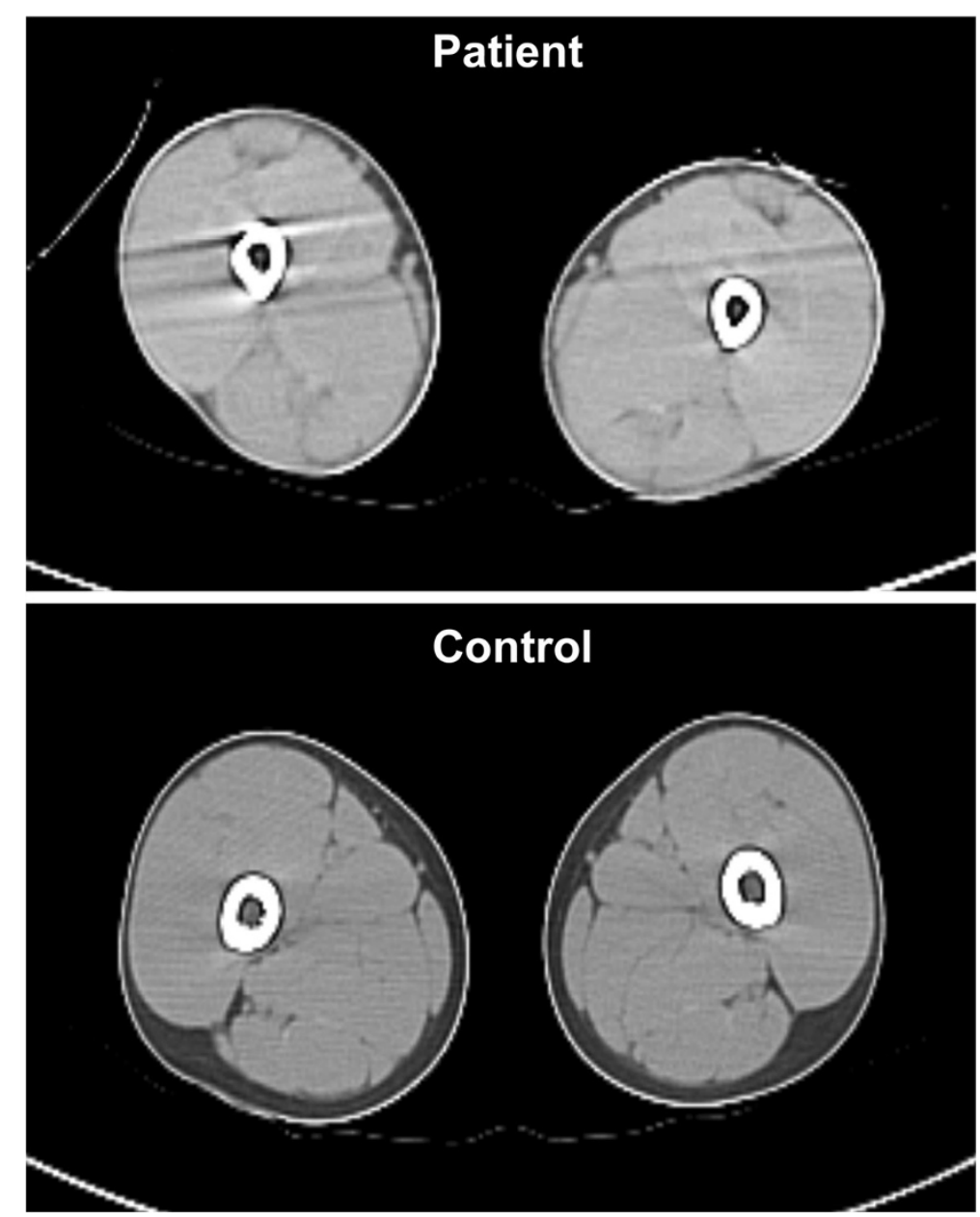

Figure 2 Lower limb CT, showing muscle hypertrophy and marked loss of subcutaneous fat.

parent and the healthy brother, and was absent in the healthy sister. The deletion was also detected in the cDNA and results in a frame shift and in introduction of the first stop codon 27 residues downstream of the correct stop codon, so the predicted protein is larger than the non-mutated protein (see ClustalW [13] predicted DNA sequence in Additional file 1: Figure S1).

Immunostaining showed that cavin-1 was absent from the sarcolemma with both antibodies used (Figure 3A). Western Blot showed absence of the band corresponding to cavin-1 (Figure 3C).

At the last visit, 14 months after diagnosis, clinical examination was unchanged with regard to the presence of generalized lypodistrophy; myopathic signs had remained mild, while fatigability and axial weakness were slightly improved and minimal acanthosis in the knees was observed.

After diagnosis the child underwent cardiological and metabolic evaluation. Echocardiogram was normal, ECG-holter monitoring showed sinus arrhythmia and few supraventricular extrasystoles, without evidence of QT prolongation. Fasting glucose, glucose tolerance test, growth hormone, cholesterol and triglyceride levels were normal. Serum leptin level was undetectable and adiponectin level was $0.83 \mu \mathrm{g} / \mathrm{ml}$ (normal 3-29 $\mu \mathrm{g} / \mathrm{ml}$ ). Finally, the patient had no history of recurrent pneumonia, and immunoglobulins were normal.

\section{Discussion}

There are few reports of PTRF gene mutations in the literature $[1-3,11,12]$. They have been found in families of Omani, UK, Japanese, Hispanic and Turkish origin. We have found a new mutation in this gene in a mildly affected child of Moroccan origin. CGL4 was not diagnosed at first, and the PTRF gene was only investigated because muscle hypertrophy was associated with marked muscle mounding and CAV3 mutations were not found. After the mutation had been detected, the patient was re-evaluated and absence of adipose tissue noted, however the lipodystrophy in this patient was mild and subtle. 


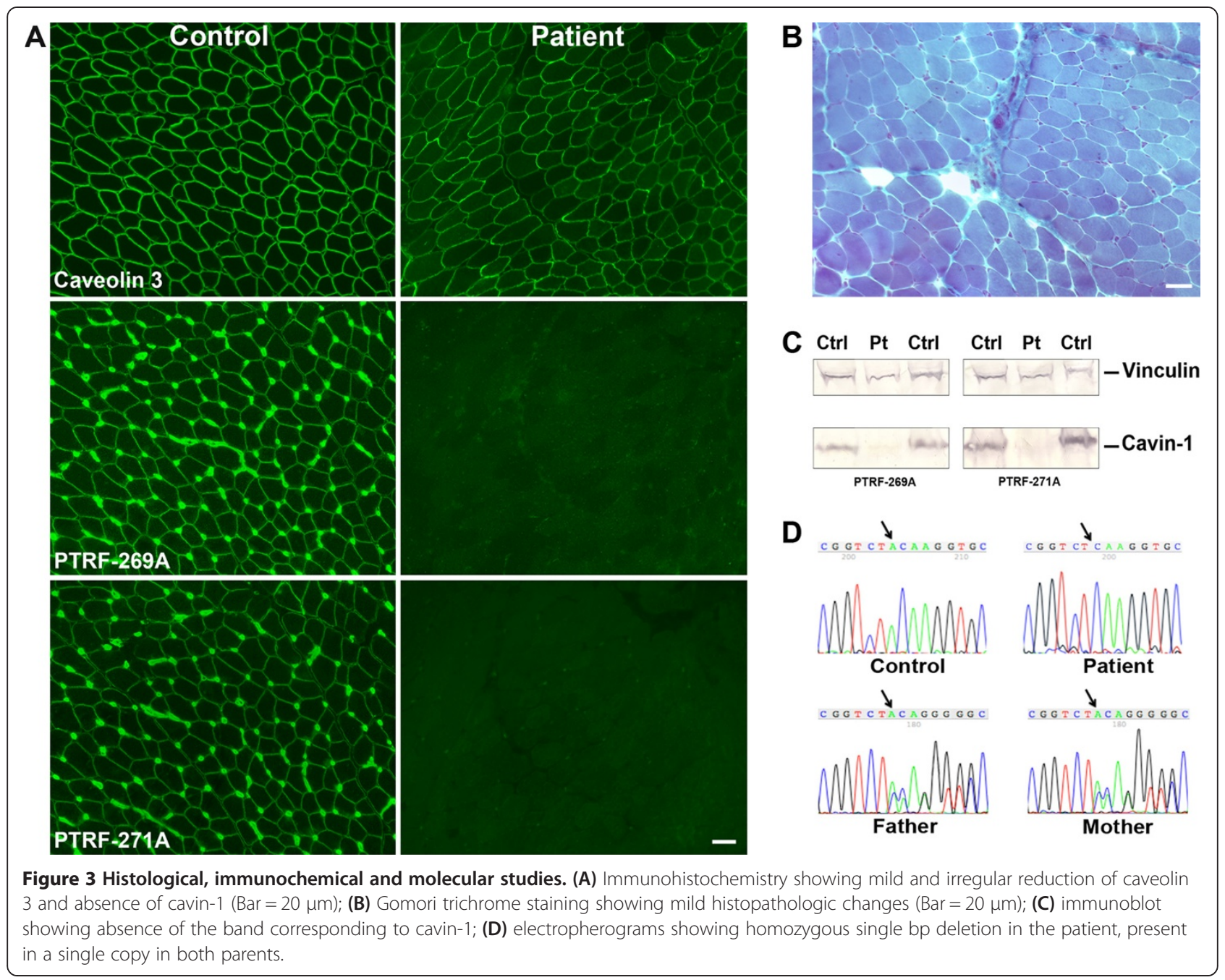

In contrast to "classic" CGL variants, symptoms are more variable in CGL4, comprising myopathy, smooth and skeletal muscle hypertrophy, cardiac arrhythmias, and skeletal abnormalities [3]. Secondary features are atlantoaxial instability, hepatomegaly, mild metabolic complications such as high circulating triglycerides and hyperinsulinism or diabetes, low leptin and adiponectin levels, reduction of growth hormone levels, immunoglobulin A deficiency, and umbilical prominence. Of these symptoms our patient had mild myopathic signs, mainly characterized by axial weakness, muscle hypertrophy, more evident in lower limbs, diffuse muscle mounding, umbilical prominence and low leptin and adiponectin levels. Other signs were absent. In particular, cardiac function was essentially normal; however the patient is still young and will require close monitoring as he grows, since the cardiac arrhythmias typical of PTRF mutated patients are potentially life-threatening: sudden deaths have been reported in consanguineous Omani families with PTRF mutations [14]. A case of similar age, but with more severe clinical features, has been recently described by Murakami et al. [12]. Differently from our patient, Murakami's case had motor delay, more severe muscle impairment, progressive metabolic abnormalities and dystrophic changes on muscle biopsy.

Although the precise mechanism of muscle rippling is unclear, some reports indicate that caveolin-3 has a role in $\mathrm{Ca}^{2+}$ homeostasis. Loss of this protein leads to microscopic disarray in the colocalization of voltagesensing dihydropyridine receptor and ryanodine receptor, thereby reducing the efficiency of excitation-contraction coupling [15].

As Cavin-1 is important in caveola formation and associates with caveolins, including caveolin-3, it is not surprising that mutations in cavin-1 cause muscle rippling or mounding.

The mutation we found affects the $\mathrm{C}$ terminus and creates a frame shift and a new stop codon 27 residues downstream of the original stop codon, producing a predicted protein of higher molecular weight. However, immunohistochemistry and Western blot indicates that 
the protein was absent from the patient's muscle, probably because it is unstable and immediately degraded.

\section{Conclusions}

To conclude, our 3-year-old boy with CGL4 had a mild muscular phenotype mainly characterized by mounding, muscle pain and hyperCKemia. We failed to detect a CAV3 mutation, but instead found a novel homozygous mutation in PTRF. Our findings suggest that, in the presence of myopathy associated with spontaneous or percussion-induced muscle contractions (rippling or mounding), mildly reduced caveolin 3 expression and no CAV3 mutation, the PTRF gene should be investigated, particularly in young patients in whom lipodystrophy may be difficult to ascertain.

\section{Consent}

Parental written informed consent was obtained for publication of this case report and any accompanying images. A copy of the written consent is available for review by the Editor of this journal.

\section{Additional file}

Additional file 1: Figure S1. Alignment of wild type and mutated PTRF gene using ClustalW2 (www.ebi.ac.uk/Tools/msa/clustalw2/). The deleted nucleotide is highlighted as well as the original stop codon and the new stop codon introduced by the mutation.

\section{Abbreviations}

CGLs: Congenital generalized lipodystrophies; CT: Computed tomography; EEG: Electroencephalography; EMG: Electromyograohy; LDH: Lactate dehydrogenase; ALT: Alanine transaminase; AST: Aspartate transaminase; MRI: Magnetic resonance imaging; MURC: Muscle-restricted coiled-coil protein; PTRF: Polymerase I and transcript release factor; SDR: Serum deprivation response; SRBC: SDR-related gene product that binds to C kinase.

\section{Competing interests}

The authors declare that they have no competing interests.

\section{Authors' contributions}

$A A, C B, M M$, IM conceived and designed the experiments and wrote the manuscript; CB carried out the molecular analysis; FB performed immunochemical evaluation of muscle biopsies and prepared the figures; SM and MLB performed biochemical studies; LM performed muscle biopsy and histological evaluation and critically revised the manuscript; AA, LC and IM performed clinical studies. All authors read and approved the final manuscript.

\section{Acknowledgments}

The authors thank Don Ward for help with the English. The study was supported by the Italian Telethon (grants n. GUP10006 to MM, GUP11001 to IM). The EuroBioBank and Telethon Network of Genetic Biobanks (GTB07001F to MM) are gratefully acknowledged for providing biological samples.

\section{Author details}

${ }^{1}$ Child Neurology Department, Fondazione IRCCS Istituto Neurologico C. Besta, Milano, Italy. ${ }^{2}$ Neuromuscular Diseases and Neuroimmunology Unit, Fondazione IRCCS Istituto Neurologico C. Besta, Milano, Italy. ${ }^{3}$ Child Neurology Unit, Ospedali Riuniti di Bergamo, Milano, Italy. ${ }^{4}$ Molecular Biology Laboratory, Istituto Auxologico Italiano IRCCS, Verbania, Italy. ${ }^{5}$ Bone Metabolism Unit, Istituto Auxologico Italiano, IRCCS, Milano, Italy. ${ }^{6}$ Muscle Cell Biology Lab, Fondazione IRCCS Istituto Neurologico "C. Besta", Via Temolo 4, 20126, Milano, Italy.
Received: 3 January 2013 Accepted: 10 September 2013

Published: 11 September 2013

\section{References}

1. Hayashi YK, Matsuda C, Ogawa M, Goto K, Tominaga K, Mitsuhashi S, Park YE, Nonaka I, Hino-Fukuyo N, Haginoya K, Sugano H, Nishino I: Human PTRF mutations cause secondary deficiency of caveolins resulting in muscular dystrophy with generalized lipodystrophy. J Clin Invest 2009, 119:2623-2633.

2. Shastry S, Delgado MR, Dirik E, Turkmen M, Agarwal AK, Garg A: Congenital generalized lipodystrophy, type 4 (CGL4) associated with myopathy due to novel PTRF mutations. Am J Med Genet A 2010, 152A:2245-2253.

3. Rajab A, Straub V, McCann LJ, Seelow D, Varon R, Barresi R, Schulze A, Lucke B, Lützkendorf S, Karbasiyan M, Bachmann S, Spuler S, Schuelke M: Fatal cardiac arrhythmia and long-QT syndrome in a new form of congenital generalized lipodystrophy with muscle rippling (CGL4) due to PTRF-CAVIN mutations. PLoS Genet 2010, 6:e1000874.

4. Hansen CG, Bright NA, Howard G, Nichols BJ: SDPR induces membrane curvature and functions in the formation of caveolae. Nat Cell Biol 2009, 11:807-814.

5. McMahon KA, Zajicek H, Li WP, Peyton MJ, Minna JD, Hernandez VJ, Luby-Phelps K, Anderson RG: SRBC/cavin-3 is a caveolin adapter protein that regulates caveolae function. EMBO J 2009, 28:1001-1015.

6. Bastiani M, Liu L, Hill MM, Jedrychowski MP, Nixon SJ, Lo HP, Abankwa D, Luetterforst R, Fernandez-Rojo M, Breen MR, Gygi SP, Vinten J, Walser PJ, North KN, Hancock JF, Pilch PF, Parton RG: MURC/Cavin-4 and cavin family members form tissue-specific caveolar complexes. J Cell Biol 2009, 185:1259-1273.

7. Nabi IR: Cavin fever: regulating caveolae. Nat Cell Biol 2009, 11:789-791.

8. Hayer A, Stoeber M, Bissig C, Helenius A: Biogenesis of caveolae: stepwise assembly of large caveolin and cavin complexes. Traffic 2010, 11:361-382.

9. Hill MM, Bastiani M, Luetterforst R, Kirkham M, Kirkham A, Nixon SJ, Walser P, Abankwa D, Oorschot VM, Martin S, Hancock JF, Parton RG: PTRF-Cavin, a conserved cytoplasmic protein required for caveola formation and function. Cell 2008, 132:113-124.

10. Liu L, Brown D, McKee M, Lebrasseur NK, Yang D, Albrecht KH, Ravid K, Pilch PF: Deletion of Cavin/PTRF causes global loss of caveolae, dyslipidemia, and glucose intolerance. Cell Metab 2008, 8:310-317.

11. Dwianingsih EK, Takeshima Y, Itoh K, Yamauchi Y, Awano H, Malueka RG, Nishida A, Ota M, Yagi M, Matsuo M: A Japanese child with asymptomatic elevation of serum creatine kinase shows PTRF-CAVIN mutation matching with congenital generalized lipodystrophy type 4. Mol Genet Metab 2010, 101:233-237.

12. Murakami N, Hayashi YK, Oto Y, Shiraishi M, Itabashi H, Kudo K, Nishino I, Nonaka I, Nagai T: Congenital generalized lipodystrophy type 4 with muscular dystrophy: clinical and pathological manifestations in early childhood. Neuromuscul Disord 2013, 23:441-444.

13. ClustalW2 - Multiple sequence alignment. http://www.ebi.ac.uk/Tools/msa/ clustalw2/.

14. Rajab A, Heathcote K, Joshi S, Jeffery S, Patton M: Heterogeneity for congenital generalized lipodystrophy in seventeen patients from Oman Am J Med Genet 2002, 110:219-225.

15. Ullrich ND, Fischer D, Kornblum C, Walter MC, Niggli E, Zorzato F, Treves S: Alterations of excitation-contraction coupling and excitation coupled $\mathrm{Ca}^{21}$ entry in human myotubes carrying CAV3 mutations linked to rippling muscle disease. Hum Mutat 2011, 32:309-317.

\section{doi:10.1186/1471-2350-14-89}

Cite this article as: Ardissone et al:: Novel PTRF mutation in a child with mild myopathy and very mild congenital lipodystrophy. BMC Medical Genetics 2013 14:89. 